\title{
Cefepime versus extended spectrum $\beta$-lactamase-producing Enterobacteriaceae
}

\begin{abstract}
The objective of this study was to evaluate the susceptibility to cefepime of a large group of ESBL- producing enterobacteria recently isolated in a Brazilian teaching hospital . The study included 280 strains of ESBL-producing enterobacteria, isolated between 2005 and 2008. The presence of the genes $b l a_{\mathrm{CTX}-\mathrm{M}}, b l a_{\mathrm{TEM}}$ and $b l a_{\mathrm{SHV}}$ was determined by PCR and confirmed by nucleotide sequencing. Susceptibility testing for cefepime was performed by disc-diffusion, agar dilution method and E-test ${ }^{\circ}$. Among the isolates, 34 (12.1\%) presented a cefepime inhibition zone $\geq 21$ and MIC $\leq 8 \mathrm{mg} / \mathrm{L}$ by agar dilution and E-strip methods. The use of cefepime for the treatment of infections caused by ESBL-producing bacteria has been controversial. Some studies of PD/PK show the probability of achieving the required PD parameters for cefepime, when the MICs were $<8 \mathrm{mg} / \mathrm{L}$, whereas others have reported therapeutic failure with the same MIC. Additional data is essential to come to terms about the report and treatment with cefepime in ESBL-producing organisms especially when these microorganisms are isolated from sterile sites and from critically ill patients.
\end{abstract}

Keywords: enterobacteriaceae; beta-lactamases; cephalosporin resistance.

[Braz J Infect Dis 2011;15(2):167-169]@Elsevier Editora Ltda.

The Clinical Laboratory Standards Institute (CLSI) has recommended, in the period of 2005 to 2009 , to perform additional tests for the detection of extended-spectrum beta-lactamases (ESBL) production among Escherichia coli, Klebsiella spp. and Proteus mirabilis isolates. Strains screened as ESBL-producers were reported as resistant to all penicillins, cephalosporins, and monobactams, regardless of the susceptibility test results. The extra step required to perform these tests and the increasing prevalence of ESBL among other Enterobacteriaceae have lead specialists to find an alternative to prevent misuse of cephalosporins. Moreover, clinical studies have demonstrated that the success of cephalosporin therapy is more related to the minimum inhibitory concentration (MIC) than to the presence of mechanisms of resistance such as the ESBL production. Based on this knowledge the European Committee on Antimicrobial Susceptibility Testing (EUCAST) and CLSI recommendations have been changed. ${ }^{1,2}$ In the CLSI guideline M100-S20 (2010), new susceptibility breakpoints were proposed for ceftriaxone $(\leq 1 \mu \mathrm{g} / \mathrm{mL})$, cefotaxime $(\leq 1 \mu \mathrm{g} / \mathrm{mL})$, ceftazi- dime $(\leq 4 \mu \mathrm{g} / \mathrm{mL})$ and aztreonam $(\leq 4 \mathrm{mg} / \mathrm{L})$, but not for cefepime $(\leq 8 \mu \mathrm{g} / \mathrm{mL}) .{ }^{1}$ According to this document, the performance of additional tests for ESBL detection would be no longer necessary except for epidemiological or infection control purposes. ${ }^{1,2}$

Cefepime, a fourth-generation cephalosporin, has been introduced into clinical practice in the mid 1990's. ${ }^{3}$ It has been recommended for treatment of Enterobacteriaceae infections as it has rapid penetration through the outer cell membrane and is stable against $\mathrm{AmpC}$ enzymes. Cefepime also demonstrates "in vitro" activity against ESBL-producing Enterobacteriaceae. In addition, the inoculum effect has not been correlated to cefepime MICs in animal models of ESBL infections. ${ }^{4}$ Studies in animal models suggest that the cephalosporin pharmacokinetic and pharmacodynamic ( $\mathrm{pK} / \mathrm{pD})$ target is similar for the treatment of ESBL- or nonESBL-producing pathogens (50\% $\mathrm{T}>\mathrm{MIC})$. Other parameters such as AUC/MIC > 1,654 and $\mathrm{C}_{\min } / \mathrm{MIC}>7.6$ also indicated good correlation with clinical cure and bacteriological eradication. According to these studies,
Authors

Keite da Silva Nogueira ${ }^{1}$ Alessandra Vale Daur ${ }^{1}$ Iara Taborda de Messias Reason ${ }^{2}$

Ana Cristina Gales ${ }^{3}$ Libera Maria Dalla Costa ${ }^{2}$

${ }^{1} \mathrm{MSc}$, Pharmacist, Hospital de Clínicas, Universidade Federal do Paraná - UFPR, Curitiba, Brazil ${ }^{2} \mathrm{PhD}$, Researcher, Hospital de Clínicas, UFPR Curitiba, Brazil ${ }^{3} \mathrm{PhD}$, Laboratório Alerta, Universidade Federal de São Paulo, São Paulo, Brazil

Submitted on: $07 / 21 / 2010$ Approved on: 08/16/2010

Correspondence to: Keite da Silva Nogueira Rua Comendador Macedo, 275. Ap.63. 80060-030 Centro, Curitiba, Paraná, Brazil keitenogueira@ hotmail.com

Financial Support: This work was supported by FUNPAR - Fundação da Universidade Federal do Paraná (FUNPAR/UFPR).

We declare no conflicts of interest. 
cefepime current therapeutic dosages would be sufficient to reach the PK/PD target. In this manner, it was not necessary to reduce cefepime susceptibility breakpoints. ${ }^{5}$

The prescription of cefepime for the treatment of ESBL infections imposes a serious clinical dilemma. On one hand, clinical use of cefepime probably would reduce carbapenem consumption. However, treatment failure with cefepime has been reported, especially, in nosocomial pneumonia due to ESBL-producing Enterobacteriaceae even in those with MICs $\leq 8 \mathrm{mg} / \mathrm{L} .{ }^{3}$ In addition, some $\mathrm{pK} / \mathrm{pD}$ studies show that the probability of achieving the $\mathrm{pK} / \mathrm{pD}$ target with cefepime is lower when the pathogen MICs varied from 1 to $8 \mathrm{mg} / \mathrm{L}$. In these cases, to achieve the desired $\mathrm{pK} / \mathrm{pD}$ target, cefepime should be prescribed in prolonged or continuous infusion regimens. ${ }^{5}$

The main objective of our study was to evaluate cefepime susceptibility in a large group of ESBL-producing Enterobacteriaceae recently isolated at a Brazilian teaching hospital.

Clinical isolates of ESBL-producing Enterobacteriaceae $(\mathrm{n}=280)$ collected from distinct body sites of patients at the Hospital of the Universidade Federal do Paraná (HC/UFPR, Curitiba, Southern Brazil) were studied. Only a single representative of a pulsed field gel electrophoresis (PFGE) pattern was included. Species identification was carried out using the VITEK system (bioMérieux, Hazelwood, MO). Susceptibility to cefepime was determined by disc-diffusion, agar dilution method and E-test ${ }^{\star}$ according to CLSI guidelines ${ }^{6}$ and manufacturer's instructions (BioMerieux, Hazelwood, $\mathrm{MO})$, respectively. ESBL encoded genes, $b l a_{\mathrm{CTX}-\mathrm{M}}, b l a_{\mathrm{TEM}}$ or $b l a_{\mathrm{SHV}}$ were analysed by polymerase chain reaction (PCR) and DNA sequencing.?

The cumulative frequency distribution of cefepime is shown in Table 1. Among 280 strains of Enterobacteriaceae, 34 (12.1\%) isolates showed cefepime inhibition zones $\geq 21 \mathrm{~mm}$ or MICs $\leq 8 \mu \mathrm{g} / \mathrm{mL}$ by disc-diffusion and agar dilution/E-test methods, respectively, and would be classified as susceptible to cefepime. They were isolated from blood (8), cerebrospinal fluid (2), peritoneal fluids (3) and urine (21) of patients hospitalized at intensive care units (12), surgical wards (9) and other clinics (13). K. pneumoniae $(64.7 \%)$ and E. cloacae (20.6\%) were the most frequent species susceptible to cefepime. Regarding the ESBL genes encoded by the Enterobacteriaceae isolates, $b a_{\mathrm{CTX}-\mathrm{M}-2}$ and $b l a_{\mathrm{SHV}-12}$ were the most frequently detected among isolates susceptible to cefepime as depicted in Figure 1.

Some studies have previously shown that extendedspectrum beta-lactamases are prevalent in many countries around the world, chiefly in South America. Using previous CLSI guidelines, all those isolates would have been considered cefepime-resistant. This recommendation leads to increased use of carbapenems and limited the use of cefepime in ESBL infections. Our study shows that $12.1 \%$ of the ESBL-producing Enterobacteriaceae tested were susceptible to cefepime. This phenotype was found most frequently in SHV-12-producing K. pneumoniae; however, a significant number of CTX-M-producing isolates, which are

Figure 1: Cefepime susceptible species and groups of ESBL.

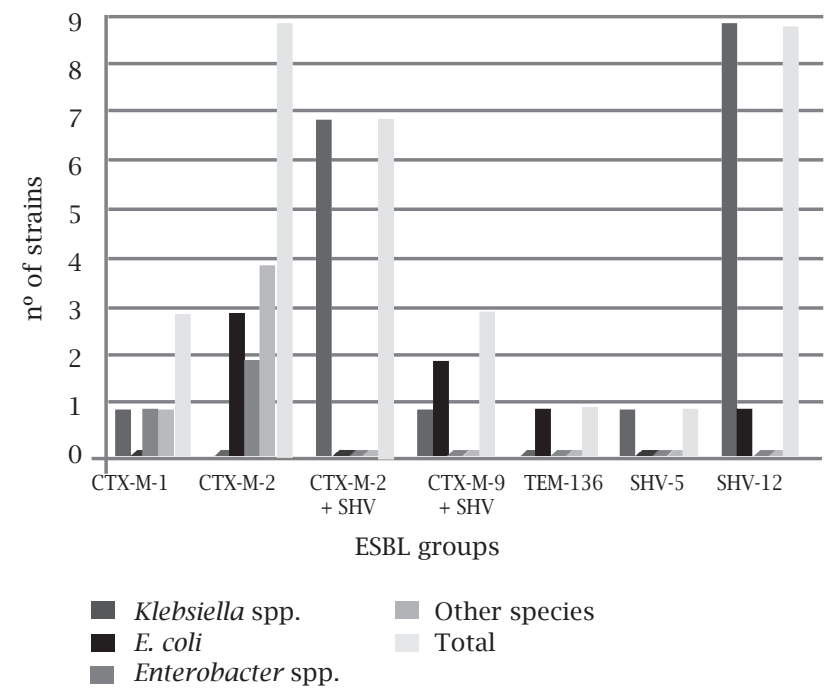

Table 1. Minimal Inhibitry concentration of cefepime in ESBL-producing enterobacteria

\begin{tabular}{|c|c|c|c|c|c|c|c|c|c|c|c|c|}
\hline \multirow[t]{2}{*}{ Microorganisms } & \multicolumn{12}{|c|}{ Percentage of isolates inhibited at cefepime MIC (mg/L) } \\
\hline & $0.06^{\mathrm{a}}$ & $0.12^{\mathrm{b}}$ & $0.25^{c}$ & $0.5^{\mathrm{d}}$ & $1.0^{\mathrm{e}}$ & $2.0^{\mathrm{f}}$ & $4.0^{\mathrm{g}}$ & $8.0^{\mathrm{h}}$ & 16.0 & 32.0 & 64.0 & $>64.0$ \\
\hline E. coli & - & - & 4.0 & 4.0 & 8.0 & 8.0 & 8.0 & 12.0 & 36.0 & 72.0 & 92.0 & 100 \\
\hline Klebsiella spp. & - & 0.8 & 1.6 & 1.6 & 4.9 & 9.8 & 13.0 & 14.6 & 33.3 & 53.7 & 74.0 & 100 \\
\hline Enterobacter spp. & 1.1 & 1.1 & 2.2 & 2.2 & 3.3 & 4.4 & 6.7 & 7.8 & 32.2 & 42.2 & 54.4 & 100 \\
\hline Others species & - & 4.8 & 4.8 & 7.1 & 9.5 & 11.9 & 14.3 & 14.3 & 45.2 & 66.7 & 71.4 & 100 \\
\hline Total & 0.36 & 1.43 & 2.50 & 2.86 & 5.36 & 8.21 & 10.71 & 12.14 & 35.0 & 53.57 & 68.93 & 100 \\
\hline
\end{tabular}

cf, cumulative frequency; MIC, minimal inhibitory concentration; --- signs the susceptible breakpoint. Letters a - h represents

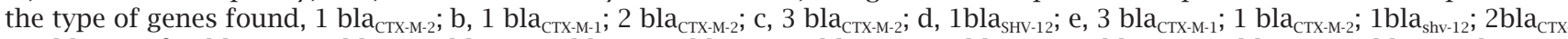

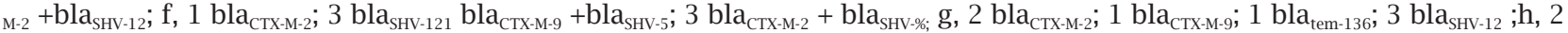
bla $_{\text {CTX-M-2 }} ; 1$ bla $_{\text {SHV-5 }} ; 1$ bla $_{\text {CTX-M-2 }}+$ bla $_{\text {SHV-5. }}$. 
the most prevalent in South America, was also susceptible to cefepime. In other geographic regions, where group TEM or SHV is prevalent, the cefepime susceptibility among ESBL-producing isolates might be even higher. In North America, $93.8 \%$ and $92.0 \%$ of the ESBL-producing E. coli and $-K$. pneumoniae, respectively, were susceptible to cefepime. ${ }^{8}$ In Taiwan, Liao et al. reported that $77 \%$ and $73.4 \%$ of the ESBL-producing E. coli and K. pneumoniae were cefepime susceptible. ${ }^{9}$

In this study, $4.8 \%$ of ESBL-producing enterobacteria isolates have shown MIC $\leq 1 \mathrm{mg} / \mathrm{L}$. PK/PD studies suggest that successful outcome using conventional regimens of cefepime ( $50 \% \mathrm{~T}>\mathrm{MIC}$ ) could be achieved for treatment of infections caused by such isolates, regardless ESBL production. ${ }^{5}$ Most authors would consider acceptable the clinical use of cefepime in these conditions. However, clinical outcomes are contradictory for infections caused by isolates, in which MICs varied from 1 to $8 \mathrm{mg} / \mathrm{L}$. For infections caused by such isolates, $\mathrm{pK} / \mathrm{pD}$ studies have demonstrated that the pharmacodynamic target could be achieved only if the infusion time or dosage were modified. ${ }^{4}$ In this manner, EUCAST recommends that only isolates exhibiting MICs for cephalosporins, including cefepime, $\leq 1 \mathrm{mg} / \mathrm{L}$ must be reported as susceptible to cephalosporins, regardless of ESBL production. ${ }^{2}$

Several reports have shown therapeutic failure when the patient had received cefepime for treatment of nonurinary infections caused by ESBL-producing organisms. These data indicate that the body site of infection might affect cefepime effectiveness. ${ }^{11}$ In our study, although most of the ESBL-producing isolates susceptible to cefepime were from urinary tract infections, $38.2 \%$ of these isolates were originated from sterile body sites of infection and $35 \%$ were from critically ill patients, increasing the chance of a possible therapeutic failure. In addition, the safety of using cefepime has been questioned. A metanalysis study associated the use of cefepime with increase in the mortality rates. ${ }^{12}$ Although this finding has been questioned by some authors, other studies have reported many untoward effects of this drug. ${ }^{3}$ Therefore, the prescription of modified cefepime regimens especially those using higher doses need more extensive evaluation before their use is encouraged.

At this moment, it would be difficult to consider cefepime a safe option for treating ESBL infections, particularly those caused by isolates with MICs between 1 and $8 \mathrm{mg} / \mathrm{L}$ or cultured from sterile sites and/or from critically ill patients. Moreover, the discordance between CLSI and EUCAST guidelines may cause confusion among microbiologists and infectious diseases specialists. While the role of cefepime in the treatment of ESBL infections is not established by clinical studies, compliance with the European guidelines seems more appropriate.

\section{REFERENCES}

1. Clinical Laboratory and Standard Institute. Performance Standards for Antimicrobial Susceptibility Testing;Twentieth Informational Supplement M100-S20. Pennsylvania: CLSI; 2010 .

2. European Committee on Antimicrobial Susceptibility Testing. Breakpoints and expert rules for $3^{\text {rd }}$ and $4^{\text {th }}$ generation cephalosporins and Enterobacteriaceae with and without beta-lactam resistance organisms. EUCAST. 2009.

3. Endimiani A, Perez F, Bonomo RA. Cefepime: a reappraisal in an era of increasing antimicrobial resistance. Expert Rev Anti Infect Ther 2008; 6:805-24.

4. Maglio D, Ong C, Banevicius MA et al. Determination of the in vivo pharmacodynamic profile of cefepime against extendedspectrum-beta-lactamase-producing Escherichia coli at various inocula. Antimicrob Agents Chemother 2004; 48:1941-7.

5. Lee, S Y, Kuti JL, Nicolau DP. Cefepime pharmacodynamics in patients with extended spectrum beta-lactamase (ESBL) and non-ESBL infections. J Infect 2007; 54:463-8.

6. Clinical Laboratory and Standard Institute. Performance Standards for Antimicrobial Disk Susceptibility Tests; approved Standard - Tenth Edition document M02-A10. Pennsylvania CLSI; 2009.

7. Payne DJ, Thomsom CJ. Molecular approaches for the detection and Identification of beta-lactamases. In: Woodford $\mathrm{N}$, Johnson AP eds. Molecular Bacteriology: Protocols and Clinical Applications. Totowa: Humana Press. 1998.

8. Sader, H S, Fritsche TR, Jones RN. Potency and spectrum trends for cefepime tested against 65746 clinical bacterial isolates collected in North American medical centers: results from the SENTRY Antimicrobial Surveillance Program (19982003). Diagn Microbiol Infect Dis 2005; 52:265-73.

9. Liao $\mathrm{CH}$, Sheng $\mathrm{WH}$, Wang JT et al. In vitro activities of 16 antimicrobial agents against clinical isolates of extended-spectrum beta-lactamase-producing Escherichia coli and Klebsiella pneumoniae in two regional hospitals in Taiwan. J Microbiol Immunol Infect 2006; 39:59-66.

10. Kotapati S, Kuti JL, Nightingale CH, Nicolau DP. Clinical implications of extended spectrum beta-lactamase (ESBL) producing Klebsiella species and Escherichia coli on cefepime effectiveness. J Infect 2005; 51:211-7.

11. Yahav D, Paul M, Fraser A, Sarid N, Leibovici L. Efficacy and safety of cefepime: a systematic review and meta-analysis. Lancet Infect Dis 2007; 7:338-48. 